\title{
The Existence of a Global Attractor for the S-K-T Competition Model with Self-Diffusion
}

\author{
Qian $\mathrm{Xu}^{1}$ and Ye Zhao ${ }^{2}$ \\ ${ }^{1}$ Department of Basic Courses, Beijing Union University, Beijing 100101, China \\ ${ }^{2}$ Department of Mathematics and Physics, Beijing Institute of Petrochemical-Technology, Beijing 102617, China
}

Correspondence should be addressed to Qian Xu; xuqian098@163.com

Received 25 January 2014; Revised 12 March 2014; Accepted 14 April 2014; Published 30 April 2014

Academic Editor: Marco Squassina

Copyright (C) 2014 Q. Xu and Y. Zhao. This is an open access article distributed under the Creative Commons Attribution License, which permits unrestricted use, distribution, and reproduction in any medium, provided the original work is properly cited.

This paper concerns the uniform bounds of the global existence of solutions in time for the S-K-T competition model with selfdiffusion. We prove that the system has a global attractor for $n<8$.

\section{Introduction and Statement of Main Result}

Shigesada et al. [1] introduced the following competition model to describe the spatial segregation of two competing species under inter- and intraspecies population pressures:

$$
\begin{array}{r}
u_{t}=\Delta\left[\left(d_{1}+\alpha_{11} u+\alpha_{12} v\right) u\right]+u\left(a_{1}-b_{1} u-c_{1} v\right), \\
x \in \Omega \subset \mathbb{R}^{n}, \quad t>0, \\
v_{t}=\Delta\left[\left(d_{2}+\alpha_{21} u+\alpha_{22} v\right) v\right]+v\left(a_{2}-b_{2} u-c_{2} v\right), \\
x \in \Omega \subset \mathbb{R}^{n}, \quad t>0, \\
\frac{\partial u}{\partial n}=\frac{\partial v}{\partial n}=0, \quad x \in \partial \Omega, t>0,
\end{array}
$$

where $\Omega$ is a bounded smooth region in $\mathbb{R}^{n}$ with $n$ as its unit outward normal vector to the smooth boundary $\partial \Omega$. $u$ and $v$ are the population densities of the two competing species. The constants $a_{j}, b_{j}, c_{j}$, and $d_{j}(j=1,2)$ are all positive, and constants $\alpha_{i j}(i, j=1,2)$ are nonnegative. $d_{1}$ and $d_{2}$ are the random diffusion rates, $\alpha_{11}$ and $\alpha_{22}$ are the self-diffusion rates which represent intraspecific population pressures, and $\alpha_{12}$ and $\alpha_{21}$ are the so-called cross-diffusion rates which represent the interspecific population pressures.

If $\alpha_{i j}=0(i, j=1,2)$, system (1) is reduced to the classical Lotka-Volterra competition model with diffusion; it has been extensively studied in the past few decades. When initial value is nonnegative and bounded, it is easy to prove that (1) has a unique uniformly bounded global solution.

For $\alpha_{11}=0$, the global existence of solutions has been widely investigated by many authors. When $n=1, d_{1}=d_{2}$, $\alpha_{12}>0, \alpha_{21}>0$, and $\alpha_{11}=\alpha_{22}=0$ hold, Kim [2] proved the global existence of classical solutions by energy method. For $n \geq 1, \alpha_{11}=\alpha_{22}=0$, Deuring [3] proved the global existence of solutions if $\alpha_{12}$ and $\alpha_{21}$ are small enough depending on the $C^{2, \alpha}$ norm of initial values $u_{0}, v_{0}$. Choi et al. [4] improved Deuring's result and proved the global existence of solutions if the cross-diffusion coefficients are small depending only on the $L^{\infty}$ norm of initial value $v_{0}$. By applying more detailed interpolated estimates, especially Gagliardo-Nirenberg inequality, Shim [5] improved Kim and Deuring's results and established the uniform bounds of the global existence of solutions in time. For $n=2$, Lou et al. [6] established the unique global existence of solutions for $\alpha_{21}=0, \alpha_{12}>0, \alpha_{11}=0$, and $\alpha_{22} \geq 0$.

For $\alpha_{11}>0,(1)$ can be written as

$$
\begin{array}{r}
u_{t}=\Delta\left[\left(d_{1}+\alpha_{11} u+\alpha_{12} v\right) u\right]+u\left(a_{1}-b_{1} u-c_{1} v\right), \\
x \in \Omega \subset \mathbb{R}^{n}, \quad t>0, \\
v_{t}=\Delta\left[\left(d_{2}+\alpha_{22} v\right) v\right]+v\left(a_{2}-b_{2} u-c_{2} v\right), \\
x \in \Omega \subset \mathbb{R}^{n}, \quad t>0,
\end{array}
$$




$$
\frac{\partial u}{\partial n}=\frac{\partial v}{\partial n}=0, \quad x \in \partial \Omega, t>0
$$

Equation (2) has been investigated by many authors; we state the results as follows.

For $n=2$, either $8 \alpha_{11}>\alpha_{12}>0,8 \alpha_{22}>\alpha_{21}>0$ or $\alpha_{22}=\alpha_{21}=0, \alpha_{11}>0$; Yagi [7] proved the global existence of solutions. For $\alpha_{11}>0, \alpha_{22}>0$, and $\alpha_{21}=0$, Kuiper and Dung [8] established the uniform bounds of global solutions for any $n$ when $\|v\|_{L^{\infty}(\Omega)}$ and $\|u\|_{L^{p}(\Omega)}(p>n)$ are uniformly bounded. Choi et al. [9] applied more detailed interpolated estimates and energy methods to prove the global existence of solutions for $n<6, \alpha_{11}>0$, and $\alpha_{22}>0$.

Le and his collaborators [10] have shown the existence of a global attractor for (2) in case $n \leq 5$. Le and Nguyen [11] constructed a special test function to prove the global existence of solutions for any dimension $n$ under some certain restrictions on coefficients. Tuôc [12] improved the results of Le and Nguyen by a nontrivial application of maximum principle. Recently, Tuoc [13] has established the $L^{4}$-estimate of $\nabla v$; then by an iteration method, they show $u \in L^{r}$ for any $r \geq 1$ and $n<10$, which implies the global existence of solutions.

In this paper, we consider the uniform bounds of the global existence of solutions in time of system (2) for $\alpha_{21}=0$, $\alpha_{11}>0$, and $\alpha_{22}>0$. In Section 2, we show some preliminary knowledge used in this paper. In Section 3, we follow the arguments of Le et al. and improve their results. We will prove the uniform bounds of the global existence of solutions in time of system (2) for $n<8$.

The main result in this paper is as follows.

Theorem 1. Assume $n<8$ holds; for any $p_{0}>n$, system (2) has a global attractor with finite Hausdorff dimension in the space $\mathscr{X}$ defined by

$$
\begin{array}{r}
\mathscr{X}=\left\{(u, v) \in W^{1, p_{0}}(\Omega) \times W^{1, p_{0}}(\Omega):\right. \\
u(x) \geq 0, \quad v(x) \geq 0, \quad \forall x \in \Omega\} .
\end{array}
$$

\section{Preliminary Results}

System (2) can be written in the divergence form as

$$
\begin{aligned}
\frac{\partial u}{\partial t}= & \nabla\left[\left(d_{1}+2 \alpha_{11} u+\alpha_{12} v\right) \nabla u+\alpha_{12} u \nabla v\right] \\
& +u\left(a_{1}-b_{1} u-c_{1} v\right), \quad x \in \Omega, t>0, \\
\frac{\partial v}{\partial t}=\nabla\left[\left(d_{2}+2 \alpha_{22} v\right) \nabla v\right] & \\
+ & v\left(a_{2}-b_{2} u-c_{2} v\right), \quad x \in \Omega, t>0, \\
& \frac{\partial u}{\partial n}=\frac{\partial v}{\partial n}=0, \quad x \in \partial \Omega .
\end{aligned}
$$

Definition 2 (see [10, Definition 2.1]). Assume that there exists a solution $(u, v)$ of system (4) defined on a subinterval
$I$ of $\mathbb{R}^{+}$. Let $\mathcal{O}$ be the set of function $\omega$ on $I$ such that there exists a positive constant $C_{0}$, which may generally depend on the parameters of the system and the $W^{1, p_{0}}$ norm of the initial value $\left(u_{0}, v_{0}\right)$, such that

$$
\omega(t) \leq C_{0}, \quad \forall t \in I .
$$

Furthermore, if $I=(0, \infty)$, one says that $\omega$ is in $\mathscr{P}$ if $\omega \epsilon$ $\mathcal{O}$ and there exists a positive constant $C_{\infty}$ that depends only on the parameters of the system but does not depend on the initial value of $\left(u_{0}, v_{0}\right)$ such that

$$
\lim _{t \downarrow \infty} \sup \omega(t) \leq C_{\infty} .
$$

If $\omega \in \mathscr{P}$ and $I=(0, \infty)$, one says $\omega$ is ultimately uniformly bounded.

Lemma 3 (the uniform Gronwall inequality). Assume that $u(t) \geq 0, a(t) \geq 0$, and $b(t) \geq 0$ hold and that they are integrable in $\left[t_{0},+\infty\right]$ satisfying

$$
\begin{gathered}
\int_{t}^{t+r} a(s) d s \leq a, \quad \int_{t}^{t+r} b(s) d s \leq b, \\
\int_{t}^{t+r} u(s) d s \leq C,
\end{gathered}
$$

where $a, b$, and $C$ are positive constants. If $u^{\prime}(t) \leq a(t) u(t)+$ $b(t)$, then one has

$$
u(t+r) \leq\left(\frac{C}{r}+b\right) e^{a}, \quad \forall t \geq t_{0} .
$$

Lemma 4 (see [10, Lemmas 3.2-3.3]). For any dimension $n$, one has the following estimates for the solutions of system (4):

$$
\begin{gathered}
\|v\|_{L^{\infty}(\Omega)} \in \mathscr{P}, \\
\|\nabla v(\cdot, t)\|_{L^{2}(\Omega)} \in \mathscr{P}, \\
\|u(\cdot, t)\|_{L^{1}(\Omega)} \in \mathscr{P}, \\
\int_{t}^{t+1} \int_{\Omega} u^{2}(x, s) d x d s \in \mathscr{P}, \\
\int_{t}^{t+1} \int_{\Omega} v_{t}^{2}(x, s) d x d s \in \mathscr{P} .
\end{gathered}
$$

Lemma 5 (see [10, Theorem 2.4]). For the system (4), if

$$
\|u\|_{q, r,[t, t+1] \times \Omega}=\left(\int_{t}^{t+1}\|u(\cdot, s)\|_{q, \Omega}^{r} d s\right)^{1 / r} \in \mathscr{P}
$$

holds, with q, r satisfying

$$
\frac{1}{r}+\frac{n}{2 q}=1-\chi, q \in\left[\frac{n}{2(1-\chi)}, \infty\right], \quad r \in\left[\frac{1}{1-\chi}, \infty\right],
$$

where $\chi \in(0,1)$, then there exists $\gamma>1$ such that

$$
\|v(\cdot, t)\|_{C^{\gamma}(\Omega)} \in \mathscr{P}, \quad\|u(\cdot, t)\|_{C^{\gamma}(\Omega)} \in \mathscr{P} .
$$




\section{Proof of Theorem 1}

Lemma 6. For any dimension $n$, any solution $u$ of (4) has the following estimate:

$$
\int_{t}^{t+1} \int_{\Omega}|\nabla v|^{4} d x d s \in \mathscr{P} .
$$

Proof. Define

$$
w=\left(d_{2}+\alpha_{22} v\right) v
$$

then $w$ satisfies the following equation:

$$
w_{t}=\left(d_{2}+2 \alpha_{22} v\right) \Delta w+\left(d_{2}+2 \alpha_{22} v\right) v\left(a_{2}-b_{2} u-c_{2} v\right) \text {. }
$$

Multiplying (19) by $\Delta w$ and integrating with respect to $x$ over $\Omega$, we have

$$
\begin{aligned}
- & \frac{1}{2} \frac{d}{d t} \int_{\Omega}|\nabla w|^{2} d x \\
= & \int_{\Omega}\left(d_{2}+2 \alpha_{22} v\right)|\Delta w|^{2} d x \\
& \quad+\int_{\Omega}\left(d_{2}+2 \alpha_{22} v\right) v\left(a_{2}-b_{2} u-c_{2} v\right) \Delta w d x .
\end{aligned}
$$

Integrating (20) over $[t, t+1]$, we obtain

$$
\begin{aligned}
& \frac{1}{2}\|\nabla w(t)\|_{L^{2}(\Omega)}^{2}-\frac{1}{2}\|\nabla w(t+1)\|_{L^{2}(\Omega)}^{2} \\
& =\int_{t}^{t+1} \int_{\Omega}\left(d_{2}+2 \alpha_{22} v\right)|\Delta w|^{2} d x d s \\
& \quad+\int_{t}^{t+1} \int_{\Omega}\left(d_{2}+2 \alpha_{22} v\right) v\left(a_{2}-b_{2} u-c_{2} v\right) \Delta w d x d s .
\end{aligned}
$$

In virtue of (9), there exist positive constants $C_{1}, C_{2}$, and $C_{3}$ such that

$$
\begin{aligned}
C_{1} \int_{t}^{t+1} \int_{\Omega}|\Delta w|^{2} d x d s \\
\leq \frac{1}{2}\|\nabla w(t)\|_{L^{2}(\Omega)}^{2}-\frac{1}{2}\|\nabla w(t+1)\|_{L^{2}(\Omega)}^{2} \\
\quad+\int_{t}^{t+1} \int_{\Omega}\left(C_{2}+C_{3} u\right)|\Delta w| d x d s
\end{aligned}
$$

Here (18) implies

$$
\nabla w=d_{2} \nabla v+2 \alpha_{22} v \nabla v
$$

By (9)-(10) and (23), we have

$$
\|\nabla w\|_{L^{2}(\Omega)} \in \mathscr{P} .
$$

Hence (22) and Hölder's inequality imply

$$
\begin{aligned}
C_{1} \int_{t}^{t+1} \int_{\Omega}|\Delta w|^{2} d x d s \leq & C_{4}+\frac{C_{1}}{2} \int_{t}^{t+1} \int_{\Omega}|\Delta w|^{2} d x d s \\
& +C_{5} \int_{t}^{t+1} \int_{\Omega}\left(C_{2}+C_{3} u\right)^{2} d x d s
\end{aligned}
$$

By (12) and (25), we get

$$
\int_{t}^{t+1} \int_{\Omega}|\Delta w|^{2} d x d s \in \mathscr{P} .
$$

Multiplying (19) by $w|\nabla w|^{2}$ and integrating with respect to $x$ over $\Omega$, we have

$$
\begin{aligned}
& \int_{\Omega} w_{t} w|\nabla w|^{2} d x \\
& =\int_{\Omega} \Delta w\left(d_{2}+2 \alpha_{22} v\right) w|\nabla w|^{2} d x+\int_{\Omega} f w|\nabla w|^{2} d x \\
& =-\int_{\Omega} \nabla w \nabla\left[\left(d_{2}+2 \alpha_{22} v\right) w|\nabla w|^{2}\right] d x+\int_{\Omega} f w|\nabla w|^{2} d x \\
& \leq-\int_{\Omega}\left(d_{2}+2 \alpha_{22} v\right)|\nabla w|^{4} d x \\
& \quad+\int_{\Omega} 2\left(d_{2}+2 \alpha_{22} v\right) w|\nabla w|^{2}\left|\nabla^{2} w\right| d x \\
& \quad-\int_{\Omega} w|\nabla w|^{2} 2 \alpha_{22} \nabla v \nabla w d x+\int_{\Omega} f w|\nabla w|^{2} d x,
\end{aligned}
$$

with $f=\left(d_{2}+2 \alpha_{22} v\right) v\left(a_{2}-b_{2} u-c_{2} v\right)$.

By (27), we get

$$
\begin{aligned}
\int_{\Omega} & \left(d_{2}+2 \alpha_{22} v\right)|\nabla w|^{4} d x \\
\leq & -\int_{\Omega} w_{t} w|\nabla w|^{2} d x+\int_{\Omega} 2\left(d_{2}+2 \alpha_{22} v\right) w|\nabla w|^{2}\left|\nabla^{2} w\right| d x \\
& -\int_{\Omega} w|\nabla w|^{2} 2 \alpha_{22}\left(\frac{\nabla w}{d_{2}+2 \alpha_{22} v}\right) \nabla w d x \\
& +\int_{\Omega} f w|\nabla w|^{2} d x .
\end{aligned}
$$

Recall that (9) and (18) yield

$$
\|w\|_{L^{\infty}(\Omega)} \in \mathscr{P} .
$$

It follows from (28) and (29) that

$$
\begin{aligned}
\int_{\Omega} & \left(d_{2}+4 \alpha_{22} v\right)|\nabla w|^{4} d x \\
\leq & C\left(\int_{\Omega}\left|w_{t}\right||\nabla w|^{2} d x+\int_{\Omega}|\nabla w|^{2}\left|\nabla^{2} w\right| d x\right) \\
& +\int_{\Omega}|f||\nabla w|^{2} d x .
\end{aligned}
$$


By Young's inequality and (30)

$$
\begin{aligned}
& d_{2} \int_{\Omega}|\nabla w|^{4} d x \\
& \leq C\left(\frac{d_{2}}{3 C} \int_{\Omega}|\nabla w|^{4} d x+C_{6} \int_{\Omega}\left|w_{t}\right|^{2} d x+\frac{d_{2}}{4 C} \int_{\Omega}|\nabla w|^{4} d x\right. \\
& \quad+C_{7} \int_{\Omega}\left|\nabla^{2} w\right|^{2} d x+\frac{d_{2}}{3 C} \int_{\Omega}|\nabla w|^{4} d x \\
& \left.\quad+C_{8} \int_{\Omega}|f|^{2} d x\right) .
\end{aligned}
$$

Since

$$
\int_{\Omega}\left|\nabla^{2} w\right|^{2} d x \leq C_{0} \int_{\Omega}|\Delta w|^{2} d x+C_{0} \int_{\Omega} u^{2} d x,
$$

together with (31), we see from (31) that

$$
\begin{gathered}
\int_{t}^{t+1} \int_{\Omega}|\nabla w|^{4} d x d s \\
\leq C\left(\int_{t}^{t+1} \int_{\Omega}\left|w_{t}\right|^{2} d x d s+\int_{t}^{t+1} \int_{\Omega}|\Delta w|^{2} d x d s\right. \\
\left.\quad+\int_{t}^{t+1} \int_{\Omega}|u|^{2} d x d s\right) \leq \widetilde{C}
\end{gathered}
$$

where $\widetilde{C}$ is independent of $t$.

Since

$$
w=\left(d_{2}+\alpha_{22} v\right) v, \quad w_{t}=d_{2} v_{t}+2 \alpha_{22} v v_{t}
$$

together with (9) and (13), we have $\int_{t}^{t+1} \int_{\Omega} w_{t}^{2}(x, s) d x d s \in \mathscr{P}$. This fact, together with (12) and (26), implies $\int_{t}^{t+1} \int_{\Omega}|\nabla w(x, s)|^{4} d x d s \in \mathscr{P}$. Hence, in view of $\nabla v=\nabla w /\left(d_{2}+2 \alpha_{22} v\right)$ and (9), we get the desired result $\int_{t}^{t+1} \int_{\Omega}|\nabla v|^{4} d x d s \in \mathscr{P}$.

Lemma 7. For any dimension n, any solution $u$ of (4) satisfies the following estimates:

$$
\|u\|_{L^{2}(\Omega)} \in \mathscr{P}, \quad\|u\|_{L^{3}(\Omega)} \in \mathscr{P} .
$$

Proof. Multiplying the first equation of (4) by $u$ and integrating, we get

$$
\begin{aligned}
& \frac{1}{2} \frac{d}{d t} \int_{\Omega} u^{2} d x \\
& =-\int_{\Omega}\left[\left(d_{1}+2 \alpha_{11} u+\alpha_{12} v\right)|\nabla u|^{2}-\alpha_{12} u \nabla u \cdot \nabla v\right] d x \\
& \quad+\int_{\Omega} u^{2}\left(a_{1}-b_{1} u-c_{1} v\right) d x
\end{aligned}
$$

Young's inequality and (36) imply

$$
\begin{aligned}
& \frac{1}{2} \frac{d}{d t} \int_{\Omega} u^{2} d x+\int_{\Omega} d_{1}|\nabla u|^{2} d x+2 \alpha_{11} \int_{\Omega} u|\nabla u|^{2} d x \\
& \quad+\alpha_{12} \int_{\Omega} v|\nabla u|^{2} d x \\
& =-\alpha_{12} \int_{\Omega} u \nabla u \cdot \nabla v d x+\int_{\Omega} u^{2}\left(a_{1}-b_{1} u-c_{1} v\right) d x \\
& \leq \varepsilon \int_{\Omega} u|\nabla u|^{2} d x+C(\varepsilon) \int_{\Omega} u|\nabla v|^{2} d x+\int_{\Omega} a_{1} u^{2} d x
\end{aligned}
$$

Taking $\varepsilon=\alpha_{11}$ in (37), we have

$$
\begin{aligned}
& \frac{1}{2} \frac{d}{d t} \int_{\Omega} u^{2} d x+\int_{\Omega} d_{1}|\nabla u|^{2} d x+\alpha_{11} \int_{\Omega} u|\nabla u|^{2} d x \\
& \quad+\alpha_{12} \int_{\Omega} v|\nabla u|^{2} d x \\
& \leq C\left(\alpha_{11}, \alpha_{12}\right) \int_{\Omega} u|\nabla v|^{2} d x+\int_{\Omega} a_{1} u^{2} d x \\
& \leq C_{9} \int_{\Omega} u^{2} d x+C_{10} \int_{\Omega}|\nabla v|^{4} d x+\int_{\Omega} a_{1} u^{2} d x
\end{aligned}
$$

By the uniform Gronwall inequality, together with (12), (17), and (38), we obtain

$$
\|u\|_{L^{2}(\Omega)} \in \mathscr{P}
$$

In virtue of (36), we have

$$
\begin{gathered}
\frac{1}{2} \frac{d}{d t} \int_{\Omega} u^{2} d x+\int_{\Omega} d_{1}|\nabla u|^{2} d x+2 \alpha_{11} \int_{\Omega} u|\nabla u|^{2} d x \\
\quad+\alpha_{12} \int_{\Omega} v|\nabla u|^{2} d x+b_{1} \int_{\Omega} u^{3} d x \\
=-\alpha_{12} \int_{\Omega} u \nabla u \cdot \nabla v d x+\int_{\Omega} u^{2}\left(a_{1}-c_{1} v\right) d x .
\end{gathered}
$$

Integrating (40) over $[t, t+1]$, we get

$$
\begin{aligned}
& \frac{1}{2}\|u(t+1)\|_{L^{2}(\Omega)}^{2}-\frac{1}{2}\|u(t)\|_{L^{2}(\Omega)}^{2}+\int_{t}^{t+1} \int_{\Omega} d_{1}|\nabla u|^{2} d x d s \\
& \quad+2 \alpha_{11} \int_{t}^{t+1} \int_{\Omega} u|\nabla u|^{2} d x d s+\alpha_{12} \int_{t}^{t+1} \int_{\Omega} v|\nabla u|^{2} d x d s \\
& \quad+b_{1} \int_{t}^{t+1} \int_{\Omega} u^{3} d x d s \\
& \leq \varepsilon \alpha_{12} \int_{t}^{t+1} \int_{\Omega} u|\nabla u|^{2} d x d s+C \int_{t}^{t+1} \int_{\Omega} u|\nabla v|^{2} d x d s \\
& \quad+\int_{t}^{t+1} \int_{\Omega} a_{1} u^{2} d x d s .
\end{aligned}
$$


By Young's inequality, we have

$$
\begin{aligned}
& \int_{t}^{t+1} \int_{\Omega} u|\nabla v|^{2} d x d s \\
& \quad \leq \frac{1}{2} \int_{t}^{t+1} \int_{\Omega} u^{2} d x d s+\frac{1}{2} \int_{t}^{t+1} \int_{\Omega}|\nabla v|^{4} d x d s
\end{aligned}
$$

Taking $\varepsilon=\alpha_{11} / \alpha_{12}$ in (41) and applying Hölder's inequality, we see from (42) that

$$
\begin{aligned}
& b_{1} \int_{t}^{t+1} \int_{\Omega} u^{3} d x d s \\
& \quad \leq \frac{1}{2}\|u(t)\|_{L^{2}(\Omega)}^{2}-\frac{1}{2}\|u(t+1)\|_{L^{2}(\Omega)}^{2} \\
& \quad+C_{11} \int_{t}^{t+1} \int_{\Omega} u^{2} d x d s+C_{12} \int_{t}^{t+1} \int_{\Omega}|\nabla v|^{4} d x d s .
\end{aligned}
$$

By (12), (17), and (39), we get

$$
\int_{t}^{t+1} \int_{\Omega} u^{3} d x d s \in \mathscr{P}
$$

Next we prove $\|u\|_{L^{3}(\Omega)} \in \mathscr{P}$. Multiplying (4) by $u^{2}$ and integrating with respect to $x$ over $\Omega$, we get

$$
\begin{aligned}
& \frac{1}{3} \frac{d}{d t} \int_{\Omega} u^{3} d x+2 \int_{\Omega} d_{1} u|\nabla u|^{2} d x+4 \alpha_{11} \int_{\Omega} u^{2}|\nabla u|^{2} d x \\
& \quad+2 \alpha_{12} \int_{\Omega} u v|\nabla u|^{2} d x \\
& =-2 \int_{\Omega} \alpha_{12} u^{2} \nabla v \cdot \nabla u d x+\int_{\Omega} u^{3}\left(a_{1}-b_{1} u-c_{1} v\right) d x
\end{aligned}
$$

Apply the following inequalities:

$$
\begin{gathered}
\int_{\Omega} v^{2} d x \leq \varepsilon\left(\int_{\Omega}|\nabla v|^{2} d x+\|v\|_{L^{1}(\Omega)}^{2}\right)+C \varepsilon^{-n / 2}\|v\|_{L^{1}(\Omega)}^{2}, \\
\int_{\Omega} u^{2} \nabla u \cdot \nabla v d x \leq \varepsilon_{1} \int_{\Omega} u^{2}|\nabla u|^{2} d x+C\left(\varepsilon_{1}\right) \int_{\Omega} u^{2}|\nabla v|^{2} d x \\
\int_{\Omega} u^{2}|\nabla v|^{2} d x \leq \frac{1}{2} \int_{\Omega} u^{4} d x+\frac{1}{2} \int_{\Omega}|\nabla v|^{4} d x
\end{gathered}
$$

Use (46) with $v=u^{2}$ to get

$$
\begin{aligned}
\int_{\Omega} u^{4} d x & \leq \varepsilon\left\{\int_{\Omega}\left|\nabla\left(u^{2}\right)\right|^{2} d x+\left\|u^{2}\right\|_{L^{1}(\Omega)}^{2}\right\}+C \varepsilon^{-n / 2}\left\|u^{2}\right\|_{L^{1}(\Omega)}^{2} \\
& =\varepsilon\left\{4 \int_{\Omega} u^{2}|\nabla u|^{2} d x+\|u\|_{L^{2}(\Omega)}^{4}\right\}+C \varepsilon^{-n / 2}\|u\|_{L^{2}(\Omega)}^{4} .
\end{aligned}
$$

Choosing small positive numbers $\varepsilon$ and $\varepsilon_{1}$ in the above inequalities, we get

$$
\begin{aligned}
& \frac{1}{3} \frac{d}{d t} \int_{\Omega} u^{3} d x+2 \int_{\Omega} d_{1} u|\nabla u|^{2} d x+\alpha_{11} \int_{\Omega} u^{2}|\nabla u|^{2} d x \\
& \quad+2 \alpha_{12} \int_{\Omega} u v|\nabla u|^{2} d x \\
& \leq C\left(\int_{\Omega} u^{2} d x\right)^{2}+C \int_{\Omega}|\nabla v|^{4} d x+\int_{\Omega} a_{1} u^{3} d x .
\end{aligned}
$$

By (17), (39), (44), (48), and uniform Gronwall's inequality, we get the desired result

$$
\|u\|_{L^{3}(\Omega)} \in \mathscr{P} .
$$

Proof of Theorem 1. It follows from (48) that

$$
\begin{aligned}
\alpha_{11} \int_{t}^{t+1} \int_{\Omega} u^{2}|\nabla u|^{2} d x d s \\
\leq \frac{1}{3}\|u(t)\|_{L^{3}(\Omega)}^{3}-\frac{1}{3}\|u(t+1)\|_{L^{3}(\Omega)}^{3} \\
\quad+C \int_{t}^{t+1}\left(\int_{\Omega} u^{2} d x\right)^{2} d s+C \int_{t}^{t+1} \int_{\Omega}|\nabla v|^{4} d x d s \\
\quad+\int_{t}^{t+1} \int_{\Omega} a_{1} u^{3} d x d s .
\end{aligned}
$$

In virtue of (17), (35), (44), and (50), we obtain

$$
\int_{t}^{t+1} \int_{\Omega} u^{2}|\nabla u|^{2} d x d s \in \mathscr{P} .
$$

For $l=2, v=u^{l}$, we see $\int_{t}^{t+1} \int_{\Omega}|\nabla v|^{2} d x d s=$ $4 \int_{t}^{t+1} \int_{\Omega} u^{2}|\nabla u|^{2} d x d s \in \mathscr{P}$.

Let $w=v-\int_{\Omega} v d x$; then Gagliardo-Nirenberg inequality gives

$$
\|w\|_{L^{2^{*}(\Omega)}} \leq C\|\nabla w\|_{L^{2}(\Omega)},
$$

which implies

$$
\|v\|_{L^{2^{*}(\Omega)}} \leq C\left(\|\nabla v\|_{L^{2}(\Omega)}+\|v\|_{L^{1}(\Omega)}\right),
$$

with $2^{*}=2 n /(n-2)$.

For $r=2 l, q=2^{*} l$, in virtue of (53), we have

$$
\begin{aligned}
\int_{t}^{t+1}\|u\|_{L^{q}(\Omega)}^{r} d s & =\int_{t}^{t+1}\|v\|_{L^{2^{*}}(\Omega)}^{2} d s \\
& \leq C\left(\int_{t}^{t+1}\|\nabla v\|_{L^{2}(\Omega)}^{2} d s+\sup _{[t, t+1]}\|v\|_{L^{1}(\Omega)}^{2}\right) .
\end{aligned}
$$

Note

$$
\|v\|_{L^{1}(\Omega)}^{2}=\|u(\cdot, t)\|_{L^{l}(\Omega)}^{l}=\|u(\cdot, t)\|_{L^{2}(\Omega)}^{2} \in \mathscr{P} ;
$$


thus $\int_{t}^{t+1}\|u\|_{L^{q}(\Omega)}^{\gamma} d s \in \mathscr{P}$, with $q$, r satisfying

$$
1-\chi:=\frac{1}{r}+\frac{n}{2 q}=\frac{1}{l}\left(\frac{1}{2}+\frac{n}{2 \cdot 2^{*}}\right)=\frac{n}{4 l} \text {. }
$$

Let

$$
A=q-\frac{n}{2(1-\chi)}=q-2 l, \quad B=r-\frac{1}{1-\chi}=2 l-\frac{4 l}{n},
$$

when $l=2$ holds; in order to satisfy (15) in Lemma 5, we need to check $\chi \in(0,1), A \geq 0$, and $B \geq 0$. By (56), we have the following results:

$$
\begin{array}{llll}
n=3 & \chi=\frac{5}{8} & A=8 & B=\frac{4}{3} \\
n=4 & \chi=\frac{1}{2} & A=4 & B=2, \\
n=5 & \chi=\frac{3}{8} & A=\frac{8}{3} & B=\frac{12}{5} \\
n=6 & \chi=\frac{1}{4} & A=2 & B=\frac{8}{3} \\
n=7 & \chi=\frac{1}{8} & A=\frac{8}{5} & B=\frac{20}{7} .
\end{array}
$$

Since $C^{v} \times C^{v}(v>1)$ is compact in $\mathscr{X}$, by the attractor theory in [14], we complete the proof of Theorem 1.

\section{Conflict of Interests}

The authors declare that there is no conflict of interests regarding the publication of this paper.

\section{Acknowledgments}

The authors would like to thank the referee for his valuable comments and helpful suggestions which helped improve the exposition of the current paper. The authors are greatly indebted to Professor Yaping $\mathrm{Wu}$ for her encouragement, valuable suggestions, and helpful discussions. This work is partially supported by Beijing Natural Science Foundation (1132003, KZ201310028030, and KM201210017008).

\section{References}

[1] N. Shigesada, K. Kawasaki, and E. Teramoto, "Spatial segregation of interacting species," Journal of Theoretical Biology, vol. 79, no. 1, pp. 83-99, 1979.

[2] J. U. Kim, "Smooth solutions to a quasi-linear system of diffusion equations for a certain population model," Nonlinear Analysis: Theory, Methods \& Applications, vol. 8, no. 10, pp. 1121$1144,1984$.

[3] P. Deuring, "An initial-boundary-value problem for a certain density-dependent diffusion system," Mathematische Zeitschrift, vol. 194, no. 3, pp. 375-396, 1987.

[4] Y. S. Choi, R. Lui, and Y. Yamada, "Existence of global solutions for the Shigesada-Kawasaki-Teramoto model with weak crossdiffusion," Discrete and Continuous Dynamical Systems, vol. 9, no. 5, pp. 1193-1200, 2003.
[5] S.-A. Shim, "Uniform boundedness and convergence of solutions to cross-diffusion systems," Journal of Differential Equations, vol. 185, no. 1, pp. 281-305, 2002.

[6] Y. Lou, W.-M. Ni, and Y. Wu, "On the global existence of a crossdiffusion system," Discrete and Continuous Dynamical Systems, vol. 4, no. 2, pp. 193-203, 1998.

[7] A. Yagi, "Global solution to some quasilinear parabolic system in population dynamics," Nonlinear Analysis: Theory, Methods \& Applications, vol. 21, no. 8, pp. 603-630, 1993.

[8] H. Kuiper and L. Dung, "Global attractors for cross diffusion systems on domains of arbitrary dimension," Rocky Mountain Journal of Mathematics, vol. 37, no. 5, pp. 1645-1668, 2007.

[9] Y. S. Choi, R. Lui, and Y. Yamada, "Existence of global solutions for the Shigesada-Kawasaki-Teramoto model with strongly coupled cross-diffusion," Discrete and Continuous Dynamical Systems, vol. 10, no. 3, pp. 719-730, 2004.

[10] D. Le, L. V. Nguyen, and T. T. Nguyen, "Shigesada-KawasakiTeramoto model on higher dimensional domains," Electronic Journal of Differential Equations, vol. 2003, no. 72, pp. 1-12, 2003.

[11] D. Le and T. T. Nguyen, "Global existence for a class of triangular parabolic systems on domains of arbitrary dimension," Proceedings of the American Mathematical Society, vol. 133, no. 7, pp. 1985-1992, 2005.

[12] P. V. Tuôc, "Global existence of solutions to shigesada-kawasakiteramoto cross-diffusion systems on domains of arbitrary dimensions," Proceedings of the American Mathematical Society, vol. 135, no. 12, pp. 3933-3941, 2007.

[13] P. V. Tuoc, "On global existence of solutions to a cross-diffusion system," Journal of Mathematical Analysis and Applications, vol. 343, no. 2, pp. 826-834, 2008.

[14] J. K. Hale, Asymptotic Behavior of Dissipative Systems, vol. 25 of Mathematical Surveys and Monographs, American Mathematical Society, Providence, RI, USA, 1988. 


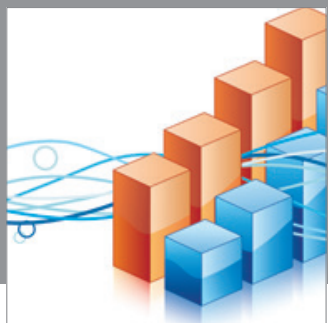

Advances in

Operations Research

mansans

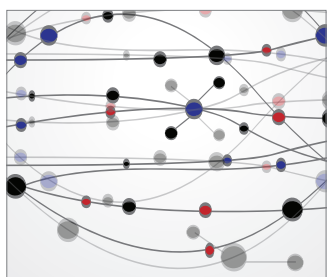

The Scientific World Journal
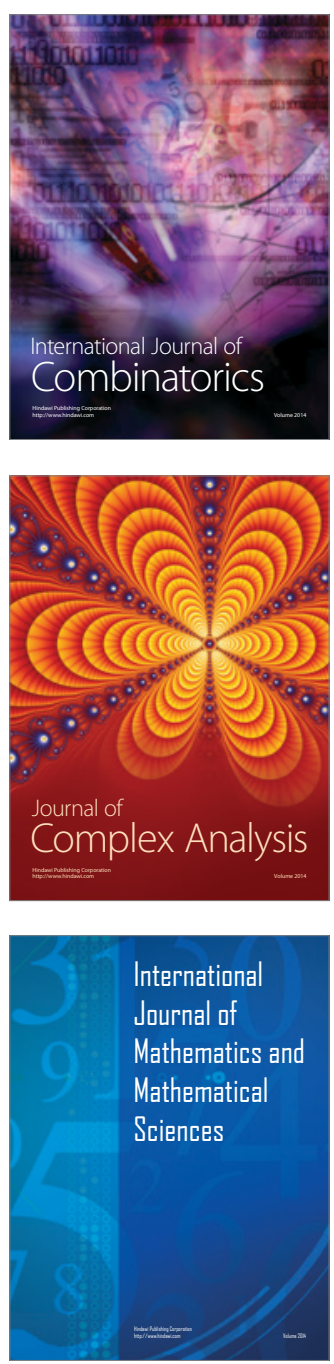
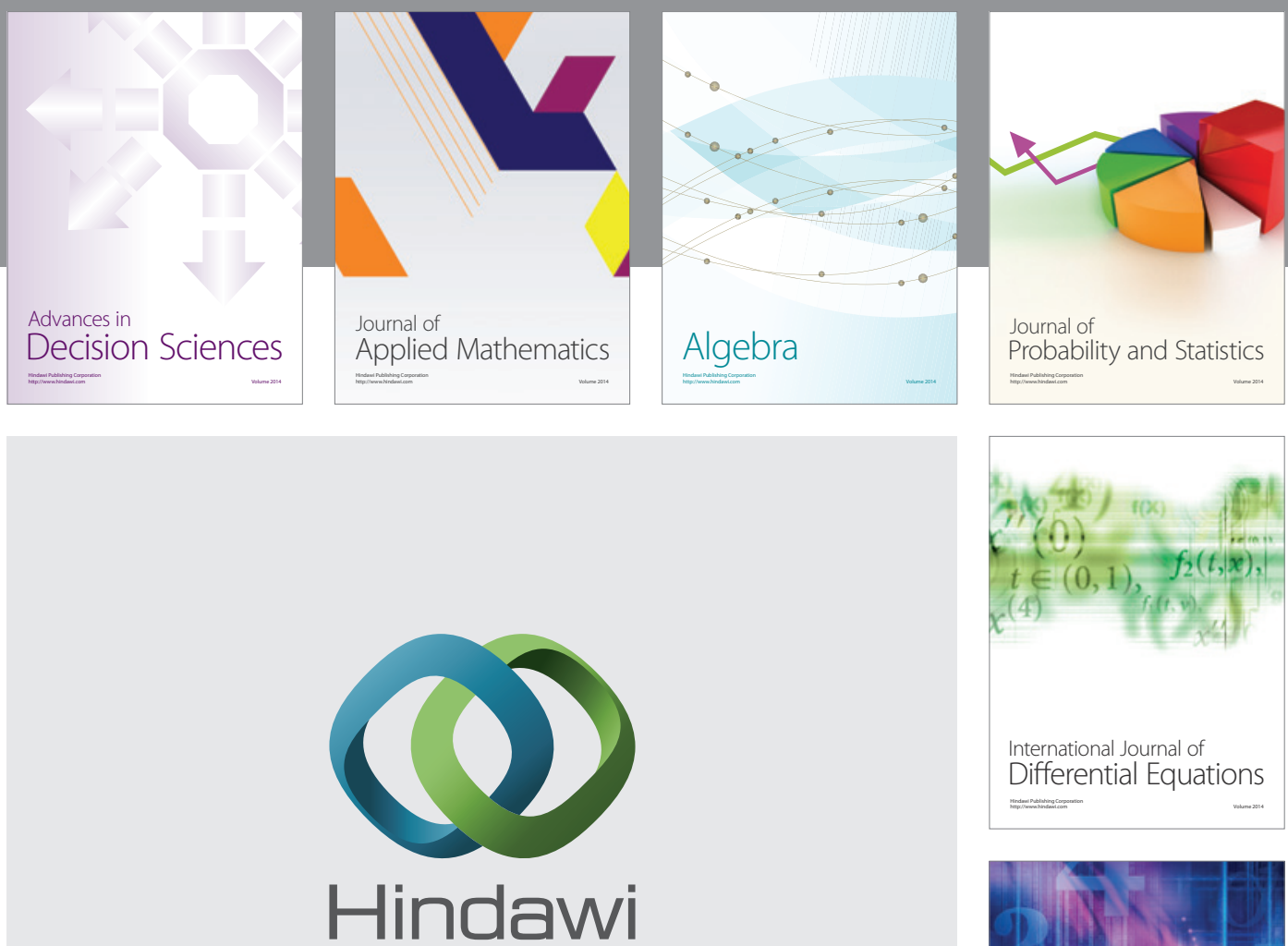

Submit your manuscripts at http://www.hindawi.com
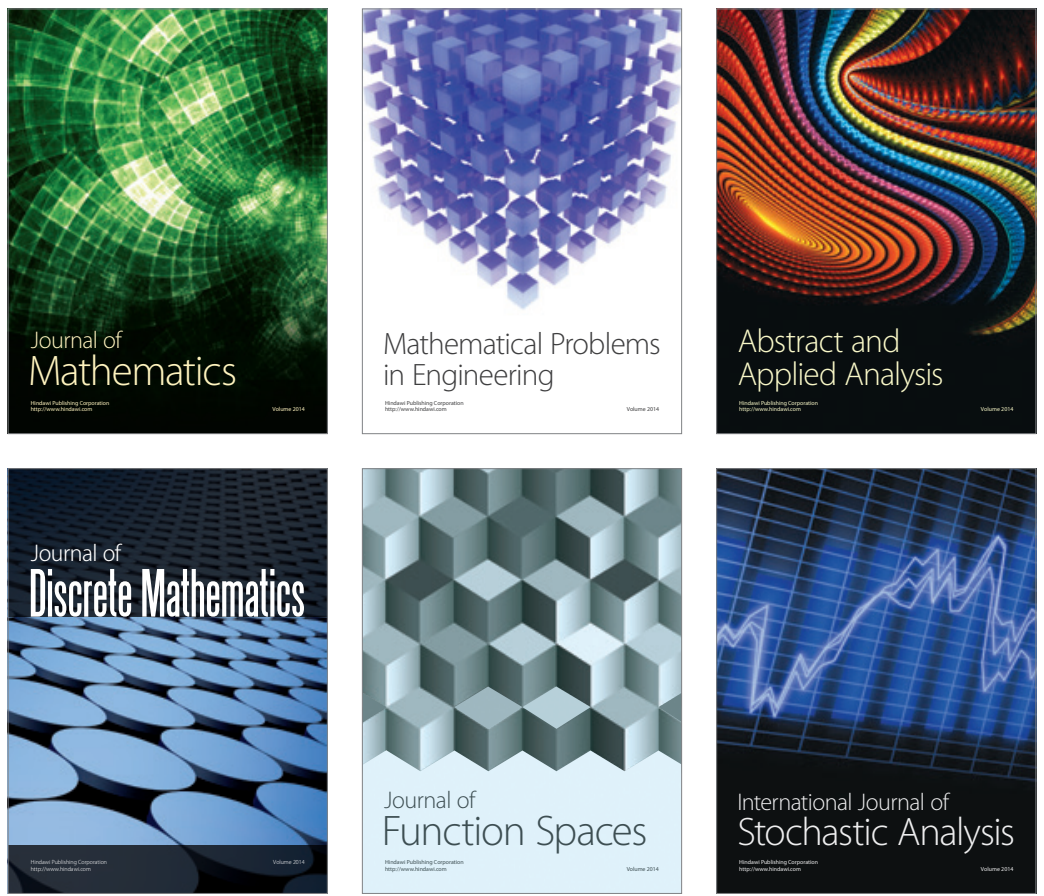

Journal of

Function Spaces

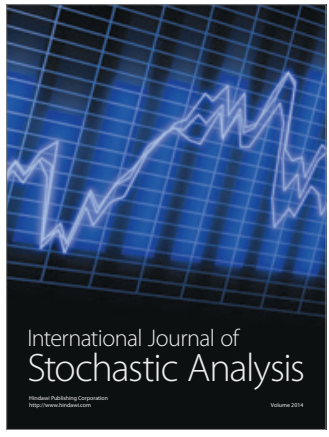

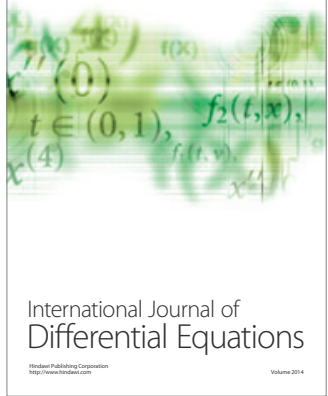
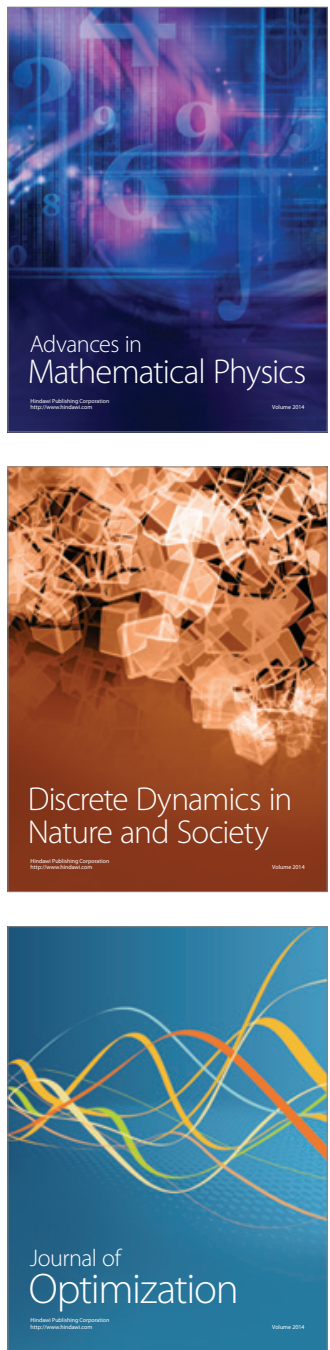